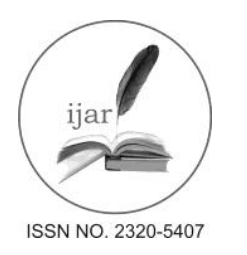

\author{
Journal homepage: http://www.journalijar.com

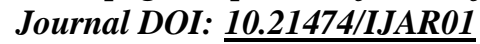

INTERNATIONAL JOURNAL

OF ADVANCED RESEARCH

RESEARCH ARTICLE

\title{
A SURVEY TOWARDS HIERARCHICAL ROUTING TECHNIQUES IN WIRELESS SENSOR NETWORKS
}

\author{
Roopa S.N $\mathbf{N}^{1}$, Chinnaswamy C.N $\mathbf{N}^{2}$. \\ 1. PG student, Department of IS\&E, The National Institute of Engineering, Mysore, India. \\ 2. Associate Professor, Department of IS\&E, The National Institute of Engineering, Mysore, India.
}

\section{Manuscript Info}

Manuscript History:

Received: 17 February 2016

Final Accepted: 22 March 2016

Published Online: August 2016

Key words:

Hierarchical based routing protocol, Cluster Head $(\mathrm{CH})$, LEACH, TLLEACH HEED, TEEN, APTEEN, and PEGASIS.

*Corresponding Author

Roopa S.N

\begin{abstract}
In recent years WSN has become evident in research areas. Wsn is a collection of several small nodes which can sense, accumulate and disseminate data for multiple applications. The sensor nodes have a limited transmission range, processing and capacity abilities and their vitality assets are additionally constrained. Routing protocols are in charge of keeping up the routes in the network and need to guarantee reliable multi hop communication in these constraints. In hierarchical architecture, nodes with higher energy can be utilized to handle and transmit the data while nodes with lower energy can be utilized to carry out the sensing in the closeness of the objective. This implies that cluster creation and assignment of special tasks to cluster heads can enormously add to generally framework versatility, lifetime, and efficiency of energy. Lower vitality utilization, by carrying out data aggregation and fusion the main goal is to diminish the number of transmitted messages to the BS which can be done by hierarchical routing. In this kind of routing there are mainly two-layer routing, one is utilized to choose the cluster heads and the latter is utilized for routing. In this paper we present a survey on these protocols as well as their merits and demerits.
\end{abstract}

Copy Right, IJAR, 2016,. All rights reserved.

\section{Introduction:-}

Wireless Sensor Networks [1] have drawn considerable measure of exploration consideration amid last decade. These networks are much disseminated systems involving two sorts of gadgets, to be specific, the sensor nodes which persistently screen their surroundings and forward the detecting information to a BS through multi-hop path, for ex- temperature sensing, infrastructure protection and environment monitoring; and the base station which is a clever remote empowered gadget responsible for accumulating the information originating from diverse sources and process them to acquire knowledge about the monitored phenomena. Sensor nodes are compact in size, less expensive radio devices with limited storage of energy, processing capacity and lifetime. Their restricted re-sources alongside the antagonistic environment put serious difficulties to the examination studies.

In numerous WSN applications, the organization of sensor nodes is accomplished in ad-hoc manner without careful arranging and designing. Once set up, the sensor nodes must have the capacity to self-organize themselves into a wireless network. Huge number of sensor nodes and their arbitrary placement in space offers redundancy in transmission of information. For the purpose of transmission radio communication are utilized as media which make them helpless to diverse sorts of assaults.

In order to secure the network furthermore its information [2], security in WSN is essential. Numerous real-time applications are in futile without confidentiality of data, integrity and availability. A secure environment which ought to handle the malicious assault is promoted by the use of security. 


\section{Challenges faced during clustering:-}

Challenges that have to be taken care during the process of cluster formation are as follows:

* Clustering cost

* Real- time operation

* Cluster and $\mathrm{CH}$ selection

* Data Aggregation

* Quality of Service (QOS)

* Synchronization

* Repair Mechanism

\section{Routing protocols:-}

The task of deploying communication paths between the nodes and later forwarding the data to the base station is done by routing.[4] Routing is challenging in WSN because of the inherent characteristics that discriminate these networks from different networks such as cellular networks. To begin with, because of the moderately huge number of sensor nodes, it is unrealistic to have a global addressing scheme for the arrangement of sensor nodes as the maintenance of ID leads to overhead. Hence IP-based protocols cannot be utilized. Second, almost all sensor networks applications requires the flow of detected data from various sources to a specific BS. This, nevertheless, does not keep the flow of data to be in different forms such as peer to peer or multicast. Third, sensor nodes are highly restricted in terms of processing, energy and capacity of storage. Hence careful management of resource is necessary. Fourth, in WSN design requirements changes with respect to applications. For instance, the testing issue of lowinertness accuracy strategic reconnaissance is not the same as that required for an occasional climate checking assignment. Fifth, awareness regarding the position of the sensor node is eminent after all collection of the data is based on location. As of now, it is not possible to utilize Global Positioning System (GPS) equipment for this purpose.

Because of the above mentioned differences, numerous algorithms are proposed in WSN for routing problem. Inherent features of WSNs alongside the application and architecture requirements are taken into consideration by the routing mechanism. Due to the restriction in energy and unexpected changes in the node status leads to successive and unusual topological changes, hence the task of discovering and maintaining routes is nontrivial in WSNs. Routing techniques put forward in literature of WSN employ familiar routing tactics, data-centric approach and tactics special to WSNS such as clustering, aggregation of data to minimize the consumption of energy.

As stated by the network structure routing protocols in WSN can be categorized as.

* Flat Network Routing,

* Hierarchical/Cluster Network Routing and

* Location-based Routing

- Based on the operation on protocol routing protocols are classified as follows

* Multipath-based,

* Query-based,

* Negotiation-based,

* QOS-based,

* Coherent and Noncoherent.

In flat networking routing protocol [5], the base station sends queries to specific region and waits for the sensors positioned in certain regions to send data. When the sensor nodes sends the sensed information to base station, intermediate nodes may carry out aggregation on the information originated from numerous sensor nodes and later forward the data towards the base station. Attribute based naming is important to state the attributes of data as the data is requested through queries. Less transmission are performed to send the data to base station hence energy can be saved.

A Natural way to deal with heterogeneous networks is hierarchical (Clustering) routing protocols [6].Heterogeneous networks are networks where few nodes have highest energy compared to other nodes. In clustering routing protocols nodes are organized to from cluster, where the information collected by nodes in a particular cluster can be aggregated and forwarded at later stages. 
In location based routing [7], locations of the nodes are used to address individual sensor nodes. Estimation of the distance between the neighboring nodes is performed by the utilization of incoming signal strength. Location of individual nodes can be accessed by a satellite through communication, via GPS if they are equipped with GPS receiver. To enhance the routing performance and to estimate the energy consumption location information can be utilized. To save energy, in some of the location based protocol the nodes are required to go to sleep state if they are not involved in any activity.

\section{Hierarchical routing protocols:-}

The entire network is partitioned into layers in hierarchical architecture [6]. In higher order level, nodes may be utilized to handle and forward the information, where as in low order level nodes are utilized to sense the data in specific regions. It can enormously add to overall scalability of the system, and the efficiency of the energy can enhance the lifetime of the network.

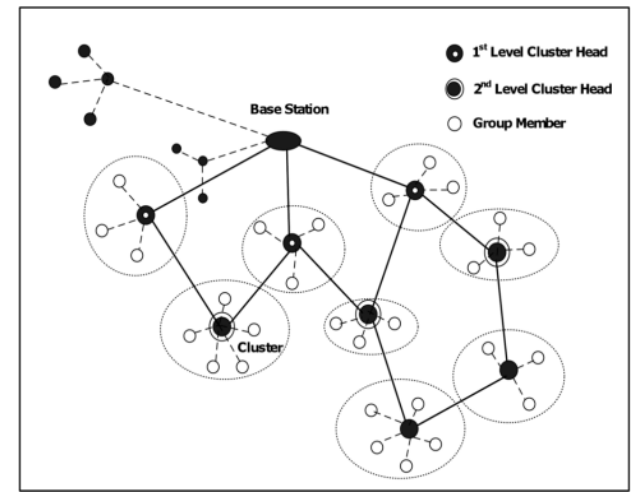

Fig: Cluster Based Model

An example of layered protocol is shown in the above fig where a network is made of many clusters and each cluster consists of a set of nodes. A cluster head is elected by the nodes in a cluster, which is responsible for managing its cluster along with the co-ordination of data transmission carried out by all the sensor nodes in it. Data is transmitted from lower layer to higher clustered layer. The data is moved faster towards the sink as it hops between layers covering a wider distance. Optimization capabilities are provided to the $\mathrm{CHs}$ through clustering.

\section{Issues in Cluster Routing Protocol:- \\ * Calculation and selection of cluster heads \\ * Scalability \\ * Topology of network \\ * Fault tolerance \\ * Redundancy management}

\section{Leach:-}

Leach Among the different clustering routing protocol leach was the initially developed protocol. LEACH [8] is an acronym that stands for Low-Energy Adaptive Clustering Hierarchy. In network, nodes are not utilized on condition that energy level is depleted. In this protocol, organization of sensor nodes as a local cluster is done by themselves where one node will be acting as a $\mathrm{CH}$. The selection of the $\mathrm{CH}$ is randomly rotated among the cluster members so that the distribution of energy load is even. Keeping in mind not to deplete the battery life of all individual nodes this randomization approach is utilized. The node makes a decision by choosing an irregular number somewhere around 0 and 1 . The decision is made based on the following threshold:

$$
T(n)=\left\{\begin{array}{c}
\frac{P}{1-P *\left(\bmod \frac{1}{P}\right)} \text { if } n \in G \\
0 \quad \text { ohterwise }
\end{array}\right.
$$


Where, $\mathrm{p}$-> cluster heads desired percentage, $\mathrm{r}$-> ongoing iteration and $\mathrm{G}->$ nodes that were not appeared as $\mathrm{CH}$ in last $1 / \mathrm{p}$ iterations.

If the number chosen in less than the threshold value, then for the ongoing iteration the node becomes a $\mathrm{CH}$. The accountability of the $\mathrm{CH}$ is the collection of data from its respective clusters and addition to that is the aggregation of the data collected to limit the number of messages forwarded to the sink, which brings about reduced energy depletion, so as to increase the lifetime of the network.

LEACH does not need global knowledge regarding the network and it's completely distributed. It utilizes single-hop routing; hence it is not suitable for networks deployed in expansive regions. The selection of the next cluster head not only requires much time but also increases the wasted of energy which result in a major drawback.

\section{TL-LEACH:-}

LEACH algorithm was further extended to TL-LEACH (Two-Level Hierarchy LEACH) [9]. Rather than using a single cluster heads, it uses the idea of two level cluster heads which are named primary and secondary cluster heads. In this technique the cluster head of each cluster, primary $\mathrm{CH}$ interacts with the secondary $\mathrm{CH}$ which in turn interacts with the sub-cluster nodes. Similar to LEACH data fusion can likewise be performed in TL-LEACH. In addition, TDMA time-slots are used to schedule the interaction within the clusters. Forwarding of data towards the sink from source nodes is done in two levels:

* From each respective cluster the secondary nodes is responsible to accumulates data from the nodes,

* Later primary $\mathrm{CH}$ in respective clusters is responsible to gather data from the secondary nodes which are then forwarded to the sink. At these individual levels data fusion can be implemented.

The major merit of this protocol is efficient reduction in the usage of energy as it decreases the amount of transmission data between the nodes and sink.

\section{HEED:-}

[10] By the utilization of residual energy, degree of a node or density as a parameter for cluster selection to accomplish power balancing it augments the basic scheme of the protocol LEACH. HEED is a multi-hop routing algorithm which aims at efficient clustering by focusing on the perfect selection of $\mathrm{CH}$, which is purely dependent on the physical distance among the node. Selection of the $\mathrm{CH}$ is dependent on the following parameters: 1) Residual energy of individual node is being utilized to select the initial group of $\mathrm{CHs}$, which is normally used as a part of numerous clustering protocols. 2) The second parameter is utilized by the nodes for the determination of cluster to which they join: Intra Cluster Communication Cost. This is useful in a situation when a given node enters into a cluster with multiple CHs. Utilization of the power level by an individual node for the announcements of intracluster and clustering is mentioned as cluster power level. Lower power levels of a cluster advances an expansion in spatial reuse where as inter cluster communication need high cluster power levels as they span multiple clusters. Accordingly, a node communicates with $\mathrm{CH}$ which yields the reduced intra-cluster communication cost during the process of cluster selection. AMRP (Average Minimum Reachability Power) measurement is used to measure the intra-cluster communication. The lifetime of the nodes is enhanced due to the evenly distribution of energy, which in turn stabilizes the neighboring node. The formation of clusters requires several iteration which leads to the broadcasting of huge packets which is a sever burden.

\section{TEEN:-}

Threshold sensitive Energy Efficient sensor Network protocol was the first protocol to be developed based on the threshold values for reactive networks, in order to react to the unexpected modification in the sensed attributes [11]. The first step involves cluster formation with each cluster drove by a $\mathrm{CH}$. The second step involves the broadcasting of two threshold values from the $\mathrm{CH}$ to its members. The threshold values are:

Hard threshold (HT): For the attributes sensed, HT is considered as the threshold value which is absolute. Nodes sensing a value beyond this must turn on its transmitter and later inform to it $\mathrm{CH}$, which in turn decreases the transmission of sensed data.

Soft Threshold (ST): It is a minor change to the hard threshold value or greater than ST value which activates the nodes to flip its transmitter and transmit the sensed data. 
This protocol is appropriately suitable for critical time applications. Application which requires periodic reports cannot adopt this kind of hierarchical protocol as the nodes stops communication with each other and the user will not receive any data regarding the network when the thresholds aren't received.

\section{APTEEN:-}

APTEEN is the augment of TEEN [12], which was introduced to overwhelm the drawback of TEEN. It is planned to procure the collection of data periodically. Similar to TEEN, transmission schedule attributes, count time and the threshold values are broadcasted by $\mathrm{CH}$. This makes feasible in capturing the data periodically. The most extreme time period among two consecutive reports interchanged by a node is said to be the count time. If the data is not sent by a node when the CT exceeds, schedule similar to TDMA is utilized and later transmission slots are assigned to the members of the cluster. APTEEN integrates both proactive methods which are similar to that of leach and reactive methods similar to TEEN. The implementation of threshold functions and count time is the main drawback of this protocol. TEEN and APTEEN performs better than LEACH.

\section{PEGASIS:-}

PEGASIS avoids the formation of cluster but in turn utilizes optimal chain based algorithm and also a data gathering algorithm [13]. These algorithm shows that conservation of energy can also be achieved by the node without cluster formation. By forming a chain structure consisting of each node and constantly collecting data over chain reduces the energy utilization. Transmission of data to the sink is done by an individual node at given time frame when chains are formed by the nodes. At each node data-fusion is carried out in the network so that all information is spread over the network. This protocol utilizes one node in the formed chain instead of multiple nodes to send data to sink. It is necessary that a node communicates only with its closest node and later those nodes alternate to communicate with sink to extend network lifetime. When all those nodes end their communication in random fashion, a new round will start to transmit the data to the sink and so on. This limits the transmission power required to send data per round as the power depleting is spread consistently among all nodes. PEGASIS accomplishes energy consumption in two different ways:

* At most two messages are received by the head node.

* Transmission of data to single hop node is done over a distance which is less.

\section{Conclusion:-}

In this paper, we have scrutinized various clustering routing protocol such as LEACH, TEEN, APTEEN, HEED, PEGASIS which are utilized in wireless Sensor Networks. This survey had a major concern to scrutinize about data aggregation, scalability, mobility, reliability, usage of power and network lifetime of all the surveyed protocols. To enhance the network lifetime, these protocol attempt to keep the sensor nodes active as long as possible which is also one of the important aspect in WSN. Number of transmitting and reception of data affects the energy utilization of individual nodes. Hence these protocols are developed such that it is energy efficient so as to enhance the lifetime of the network.

\section{Reference:-}

1. Sanjeev Kumar Gupta, Poonam Sinha "Overview of Wireless Sensor Network: A Survey", International Journal of Advanced Research in Computer and Communication Engineering Vol. 3, Issue 1, January 2014.

2. Mr. Manish M Patel, Dr. Akshai Aggarwal," Security Attacks in Wireless Sensor Networks: A Survey" 2013 International Conference on Intelligent Systems and Signal Processing (ISSP).

3. K Kalaivani, and G Indhumathi, "A Survey: Clustering Routing Algorithms for Isolated Nodes Using Wireless sensor network “, ISSN: 0975-8585, January-February 2016 RJPBCS 7(1) Page No. 862

4. Jyoti Kumari, Prachi, "A Comprehensive Survey of Routing Protocols in Wireless Sensor Networks", 2015 2nd International Conference on Computing for Sustainable Global Development (INDIA Com)

5. Jamal N. AI-Karaki, Ahmed E. Kamal, "Routing Techniques in Wireless Sensor Networks: A Survey", Dept. of Electrical and Computer Engineering, Iowa State University, Ames, Iowa 500Il

6. Twinkle Tiwari, Nihar Ranjan Roy," Hierarchical Clustering in Heterogeneous Wireless Sensor Networks: A Survey", International Conference on Computing, Communication and Automation (ICCCA2015)

7. Shital Y. Agrawal, Prof. C. M. Raut," A Survey on Location Based Routing Protocols for Wireless Sensor Network", International Journal of Emerging Technology and Advanced Engineering, Volume 3, Issue 9, September 2013. 
8. T.Kiruthika, M.Indira," A Survey on Leach Routing Protocol for Wireless Sensor Network", International Journal of Advanced Research in Computer Engineering \& Technology (IJARCET) Volume 4 Issue 1, January 2015

9. V. Loscri, G. Morabito and S. Marano, "A two-levels hierarchy for low-energy adaptive clustering hierarchy (TL-LEACH)," in Proc. IEEE 62nd Vehicular Technology Conference, 2005.

10. Ossama Younis and Sonia Fahmy, Heed: A hybrid, Energy efficient, Distributed Clustering Approach for Adhoc Networks, IEEE Transactions on Mobile Computing, vol. 3, no. 4, pp. 366-369, Oct.-Dec. 2004.

11. Manjeshwar A, Agrawal D.TEEN:A Protocol for Enhanced Efficiency in Wireless Sensor Networks[C]. In Proceeding of the 1th International Workshop on Parallel and Distributed Computing Issues in Wireless Networks and Mobile Computing'01, 2001 : 2009-2015.

12. Manjeshwar A,Agrawal D. APTEEN:A Hybrid Protocol for Efficient Routing and Comprehensive Information Retrieval in Wireless Sensor Networks[C].In Proceeding of the 2nd International Workshop on Parallel and Distributed Computing Issues in Wireless Networks and Mobile Computing'02,2002:195-202.

13. Lindse S, Raghavendra C.PEGASIS: Power-efficient Gatgering in Sensor Information Systems[C].In proceeding of the IEEE Aerospace Conference. Montana: IEEE Aerospace and Electronic System Society, 2002:1125-1130. 